University of Nebraska - Lincoln

DigitalCommons@University of Nebraska - Lincoln

John R. Hardy Papers

Research Papers in Physics and Astronomy

3-15-1970

\title{
Calculations of the Intensity of X-Ray Diffuse Scattering Produced by Point Defects in Cubic Metals
}

John W. Flocken

University of California, Livermore, California

John Hardy

University of Nebraska - Lincoln

Follow this and additional works at: https://digitalcommons.unl.edu/physicshardy

Part of the Physics Commons

Flocken, John W. and Hardy, John, "Calculations of the Intensity of X-Ray Diffuse Scattering Produced by Point Defects in Cubic Metals" (1970). John R. Hardy Papers. 23.

https://digitalcommons.unl.edu/physicshardy/23

This Article is brought to you for free and open access by the Research Papers in Physics and Astronomy at DigitalCommons@University of Nebraska - Lincoln. It has been accepted for inclusion in John R. Hardy Papers by an authorized administrator of DigitalCommons@University of Nebraska - Lincoln. 


\title{
Calculations of the Intensity of X-Ray Diffuse Scattering Produced by Point Defects in Cubic Metals*
}

\author{
JOHN W. FLOCKEN† AND JOHN R. HARDY $\ddagger$ \\ Lawrence Radiation Laboratory, University of California, Livermore, California 94550
}

(Received 28 July 1969)

\begin{abstract}
We have calculated isointensity profiles for the diffuse $x$-ray scattering associated with certain types of defects in $\mathrm{Cu}, \mathrm{Al}, \mathrm{Na}, \mathrm{K}, \mathrm{Li}$, and a theoretical model lattice. These profiles were computed for high-symmetry planes very close to reciprocal-lattice points of the $(S, 0,0),(S, S, 0)$, and $(S, S, S)$ type. Both cubic and double-force defects were treated. The calculations were done using a technique presented by Kanzaki for the theoretical model lattice. Kanzaki's general conclusion that cubic defects produce leminiscate profiles and that double-force defects produce ellipsoidal profiles is confirmed for all the material studied. Our profiles for the model lattice agree with those obtained by Kanzaki, except for the profiles due to a double-force defect near an $(S, S, S)$ reciprocal-lattice point.
\end{abstract}

\section{INTRODUCTION}

W HEN a defect is introduced into a crystal, it causes the atoms of the host lattice to become displaced from their perfect lattice sites to new equilibrium positions. These strain-field effects produce an associated change in the crystal volume, a change in its macroscopic electrical resistivity, and a diffuse x-ray scattering superimposed on the Bragg peaks of the normal lattice. Of these three effects, the last contains the most detailed information about the structure of the defect and can be used to discriminate between point defects and defect aggregates.

Kanzaki ${ }^{1}$ presented a method for calculating the isointensity contours of the x-ray diffuse scattering associated with certain classes of defects. In particular, he applied the method to defects in an fcc model lattice, the elastic constants of which satisfied the constraint that $C_{11}=2 C_{12}=2 C_{44}$. The technique used in per-

* Work performed under the auspices of the U. S. Atomic Energy Commission.

† Summer employee, Lawrence Radiation Laboratory, Livermore, Calif. 94550. Permanent address: Physics Laboratory, University of Nebraska at Omaha, Omaha, Neb. 68132.

t Summer visiting Professor of Physics, Lawrence Radiation Laboratory, Livermore, Calif. 94550. Permanent address: Behlen Laboratory of Physics, University of Nebraska, Lincoln, Neb. 68508 .

${ }_{1}^{1}$ H. Kanzaki, J. Phys. Chem. Solids 2, 107 (1957). forming these calculations is an application of the method of lattice statics. ${ }^{2-4}$ This method is based on the Fourier transformation of the direct-space equilibrium equations for a lattice containing $N$ host atoms and one defect, which is taken to be at the center of the crystal. Periodic boundary conditions are imposed across the face of the crystal, and this can be shown to be equivalent to solving the problem for a superlattice of defects with one defect in each supercell. Each supercell contains $N$ atoms. The Fourier transformation reduces the $3 N \times 3 N$ matrix of direct-space equilibrium equations to $N$-independent $3 \times 3$ matrix equations, each of which determines one of the Fourier amplitudes of the displacement field. Each of these equations is readily soluble and one then determines the direct-space displacements by Fourier inversion. However, as we show, this technique is particularly suited to the study of diffuse $\mathrm{x}$-ray scattering, since it is the Fourier amplitudes themselves which enter directly into the relevant equations. In the present paper, we are only concerned, as was Kanzaki, with the diffuse scattering in the immediate vicinity of the Bragg peaks, and this enables us to use the lattice-statics equations appropriate to

${ }^{2}$ H. Kanzaki, J. Phys. Chem. Solids 2, 24 (1957).

${ }^{3}$ J. R. Hardy, J. Phys. Chem. Solids 15, 39 (1960).

${ }_{4}^{4}$ J. W. Flocken and J. R. Hardy, Phys. Rev. 175, 919 (1968). 
FIG. 1. Isointensity profiles near an $(S, 0,0)$ reciprocal-lattice point $(\mathrm{A})$ for a cubic defect, and (B) for a doubleforce defect along $\langle 110\rangle$, in a model lattice $\left(C_{11}=2 C_{12}=2 C_{44}\right)$. Solid lines, in the [001] plane; dashed lines, in the [01ī] plane (arbitrary units).

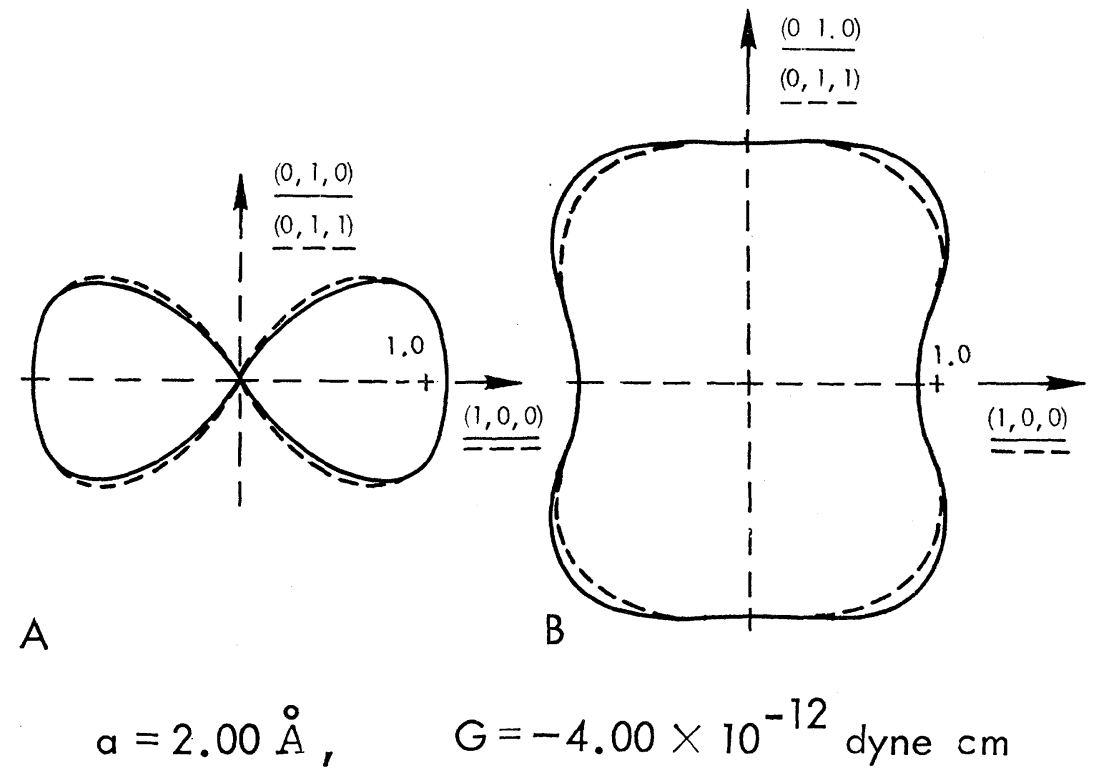

small wave vectors $\mathbf{q}$ where, as we have shown in an earlier paper, ${ }^{5}$ the Fourier amplitudes are determined by the elastic constants and the symmetry and strength of the defect.

The diffuse scattering of $x$ rays by defects has been studied and developed extensively in recent years and a complete review of the subject has been given by Keating ${ }^{6}$ and applied by Goland and Keating. ${ }^{7}$ The present work is not intended to be as comprehensive as these recent publications. We concentrate our attention on what they have termed "Huang scattering" which is, in fact, the diffuse scattering treated by
Kanzaki. However, the expression for this scattering assumes a particularly simple form when we make use of the Fourier transformations of the displacement field inherent in the theory of lattice statics, and, as we show, we can compute the Huang scattering exactly for cubic materials of arbitrary anisotropy. We are thus not restricted, as Goland and Keating were, to considering a purely isotropic medium.

We have repeated Kanzaki's calculations and agree with his results, except for the scattering intensity profiles about $(S, S, S)$-type reciprocal-lattice points in the presence of a single double-force defect. As pre-

FIG. 2. Isointensity profiles near an $(S, S, 0)$ reciprocal-lattice point $(\mathrm{A})$ for a cubic defect, and (B) for a doubleforce defect along $\langle 110\rangle$, in a model lattice $\left(C_{11}=2 C_{12}=2 C_{44}\right)$. Solid lines, in the [001] plane; dashed lines, in the [110] plane (arbitrary units).

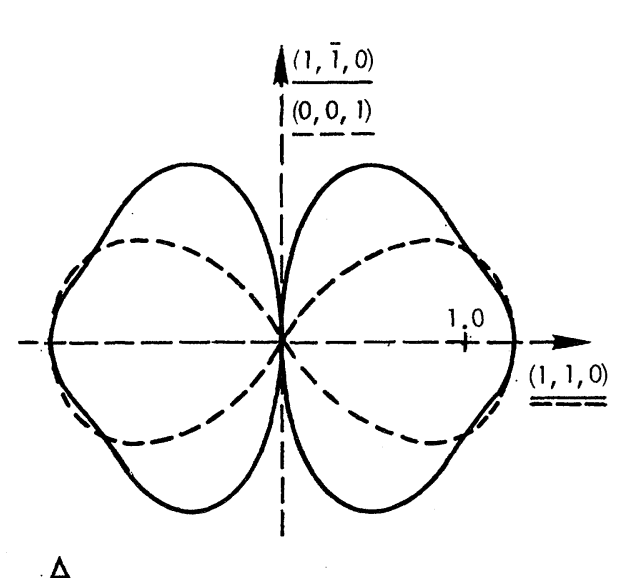

A

$$
a=2.00 \AA, \quad G=-4.00 \times 10^{-12} \text { dyne } \mathrm{cm}
$$

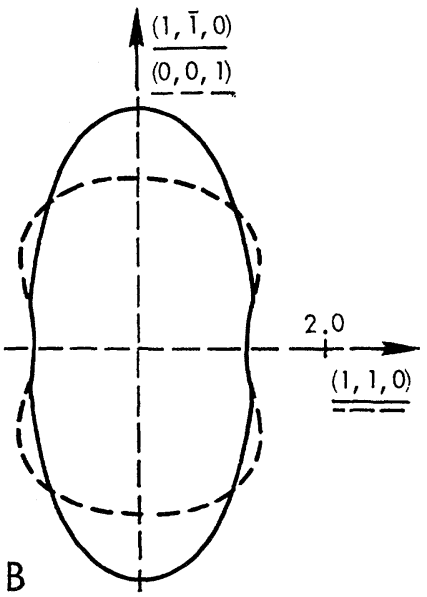

${ }^{5}$ J. W. Flocken and J. R. Hardy, Lawrence Radiation Laboratory Report No. UCRL-71516, 1969 (unpublished).

6 D. T. Keating, J. Phys. Chem. Solids 29, 771 (1968).

7 A. N. Goland and D. T. Keating, J. Phys, Chem. Solids 29, 785 (1968). 


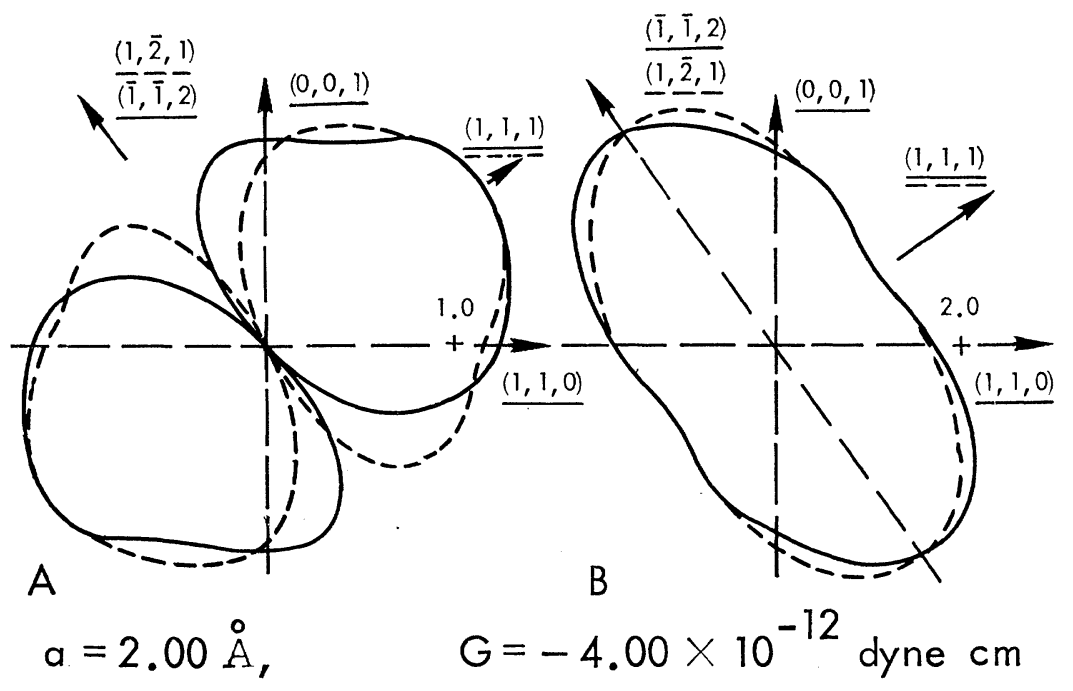

FIG. 3. Isointensity profiles near an $(S, S, S)$ reciprocal-lattice point (A) for a cubic defect, and (B) for a doubleforce defect along $\langle 110\rangle$, in a model lattice $\left(C_{11}=2 C_{12}=2 C_{44}\right)$. Solid lines, in the [110] plane; dashed lines, in the $[10 \overline{1}]$ plane.

dicted by Kanzaki, we find that spherical defects give rise to intensity profiles of the lemniscate type, while double-force defects produce ellipsoidal profiles.

We have performed the same calculations for cubic and double-force defects in $\mathrm{Cu}, \mathrm{Al}, \mathrm{Na}, \mathrm{K}$, and $\mathrm{Li}$. Although there are distinct variations in the intensity profiles among the various materials as compared with those for Kanzaki's model crystal. the profiles for the cubic defects are always of the lemniscate type and those due to double forces are always ellipsoidal.

In Sec. II, we discuss briefly the derivation of the equation for the amplitude of the defect-induced diffuse scattering and restate the form of the asymptotic displacement equations that we have given in a previous paper $^{5}$ and use in our present calculations. In Sec. III, we discuss these calculations and present the resulting isointensity profiles. Section IV is devoted to a discussion of our results and in Sec. V, we summarize the present work.

\section{DIFFUSE X-RAY SCATTERING AMPLITUDE}

A complete derivation of the $\mathrm{x}$-ray scattering amplitude in an imperfect lattice was presented in Kanzaki's original paper. ${ }^{1}$ Here we only review briefly that portion of the derivation which pertains to our calculations. The displacements, $\xi\left(\mathbf{r}^{l}\right)$ of an atom at the perfect lattice position $\mathbf{r}^{l}$, is given by

$$
\xi\left(\mathbf{r}^{l}\right)=\frac{1}{N} \sum_{\mathbf{q}} \mathbf{Q}(\mathbf{q}) \exp \left(i \mathbf{q} \cdot \mathbf{r}^{l}\right),
$$

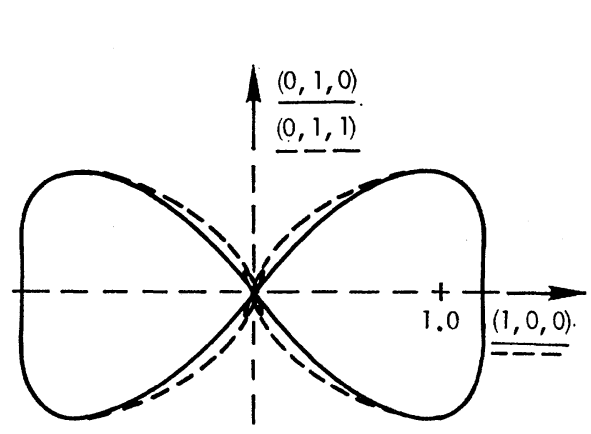

A

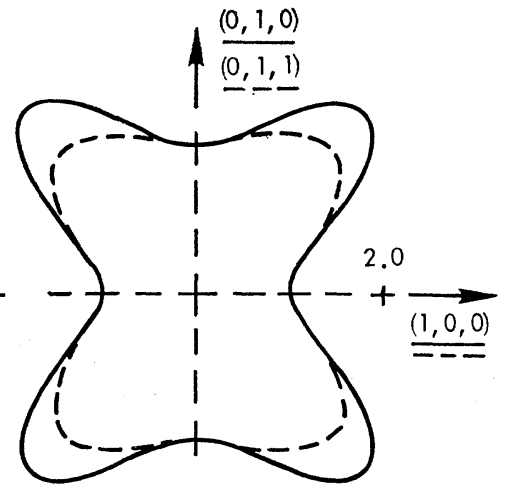

B
FIG. 4. Isointensity profiles near an $(S, 0,0)$ reciprocal-lattice point $(\mathrm{A})$ for a cubic defect, and (B) for a doubleforce defect along $\langle 110\rangle$, in $\mathrm{Cu}$. Solid lines, in the [001] plane; dashed lines, in the [011] plane.

$$
\begin{aligned}
& a=1.805 \AA \quad G=-3.89 \times 10^{-12} \text { dyne } \mathrm{cm} \\
& C_{11}=1.70 \times 10^{12} \text { dynes } / \mathrm{cm} \\
& C_{12}=1.23 \times 10^{12} \text { dynes } / \mathrm{cm} \\
& C_{44}=0.75 \times 10^{12} \text { dynes } / \mathrm{cm}
\end{aligned}
$$


FIG. 5. Isointensity profiles near an $(S, S, 0)$ reciprocal-lattice point $(\mathrm{A})$ for a cubic defect, and (B) for a doubleforce defect along $\langle 110\rangle$, in $\mathrm{Cu}$. Solid lines, in the [001] plane; dashed lines in the $[1 \overline{1} 0]$ plane.

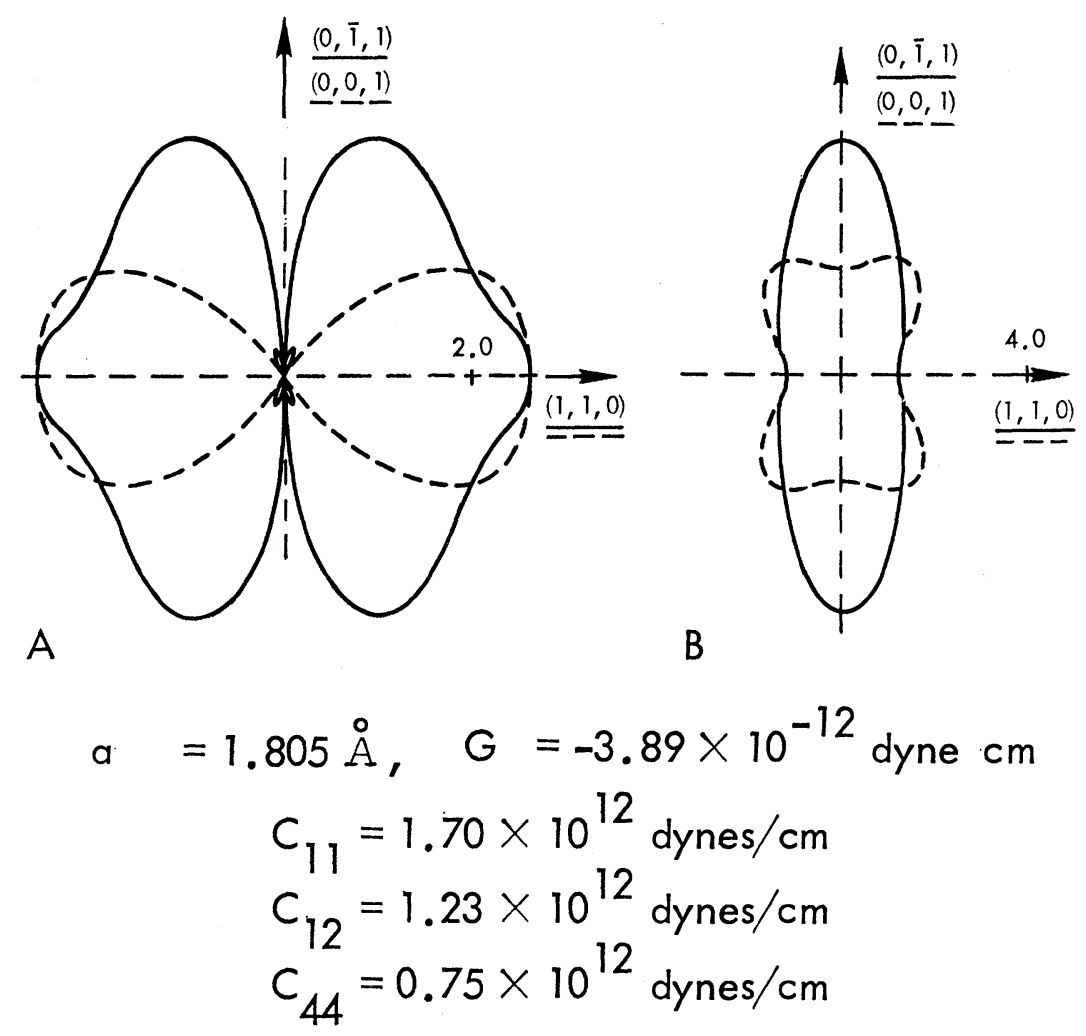

in which the sum is taken over all distinct wave vectors $\mathbf{q}$ in the first Brillouin zone. The $\mathbf{Q}(\mathbf{q})$ are the appropriate Fourier amplitudes, which are obtained from the equation

$$
\mathbf{Q}(\mathbf{q})=\left(\mathbf{V}^{-\mathbf{q}}\right)^{-1} \mathbf{F}^{\mathbf{q}},
$$

where $\mathrm{V}^{-\mathrm{q}}$ is the Fourier-transformed force-constant matrix and $\mathbf{F}^{\mathrm{q}}$ is the Fourier-transformed force array appropriate to the particular defect we are considering.

If the scattering is weak, as we expect, the scattering amplitude is given by

$$
A_{\mathrm{se}}=\sum_{l} f(l) \exp \left\{i \mathbf{K} \cdot\left[\mathrm{r}^{l}+\xi\left(\mathbf{r}^{l}\right)\right]\right\} .
$$

Here $f(l)$ is the atomic scattering factor of the atom at $\mathbf{r}^{l}$ and $\mathbf{K}$ is the scattering vector $=\mathbf{k}_{\text {in }}-\mathbf{k}_{\text {out }}$, where these are the appropriate incident and scattered $\mathrm{x}$-ray wave vectors. We neglect any contribution to the diffuse scattering arising from the defect itself, and thus confine ourselves to the strain-field scattering alone. This can be shown to be a good approximation in the immediate vicinity of the Bragg peaks, which is the region of reciprocal space that we are discussing in the present paper. It should be stressed that our present results are strictly valid for a single defect, but can plausibly be used for finite but small concentrations of randomly distributed defects. In this limit, one simply multiplies the scattering amplitude for one defect by the total number of defects present.

For low-order diffraction, where $\mathbf{K}$ is the same order of magnitude as an inverse lattice constant, the scat- tering amplitude in Eq. (2) may be expanded as a power series in $\mathbf{K} \cdot \xi^{l}\left(\mathbf{r}^{l}\right)$; thus,

$$
\begin{aligned}
A_{\mathrm{sc}}=f_{0} \sum_{l}\left\{\exp \left(i \mathbf{K} \cdot \mathbf{r}^{l}\right)[1+\right. & i \mathbf{K} \cdot \xi\left(\mathbf{r}^{l}\right) \\
& \left.\left.-\frac{1}{2}\left(\mathbf{K} \cdot \xi\left(\mathbf{r}^{l}\right)\right)^{2}+\cdots\right]\right\},
\end{aligned}
$$

where $f_{0}$ is the scattering factor of the host atoms. The first term in the square brackets simply gives rise to the normal Bragg scattering. The second term is responsible for the defect diffuse scattering, and the third term can be shown to give rise to the diminution of the intensity of the Bragg peaks due to the existence of the diffuse scattering. Henceforth, we shall discuss only the second term, relating to the defect diffuse scattering. ${ }^{8}$ Substituting for $\xi^{l}$ from Eq. (1) into Eq. (3) we obtain, for the diffuse-scattering amplitude,

$$
A_{\mathrm{ds}}=i f_{0} \mathbf{K} \sum_{\mathbf{q}} \mathbf{Q}(\mathbf{q}) \cdot \sum_{l} \exp \left[i(\mathbf{K}+\mathbf{q}) \cdot \mathbf{r}^{l}\right]
$$

${ }^{8}$ This simplification leads to the omission of two terms which, in general, will contribute to the diffuse-scattering intensity. One of these is a term of the form $i \mathbf{K} \cdot \xi^{l} e^{i \mathbf{K} \cdot r^{l}}$ which is related to the sizes and scattering factors of the defects relative to the host atoms. Since many of the defects treated in our present calculations are generalizations represented by triple double forces or single double forces we have not attempted to evaulate this "size-effect" contribution. In addition, we are omitting a term of the form $\left[\mathbf{K} \cdot \xi\left(\mathbf{r}^{l}\right)\right]^{2} e^{i \mathbf{K} \cdot r l}$ which, within the Kanzaki approximation, simply gives a diminution of the intensity of the Bragg peaks. If we allow for the difference between the scattering factor of the defect and the scattering factor of the atoms of the host lattice, these terms can also contribute to the diffuse scattered intensity. 
But

$$
\sum_{l} \exp \left[i(\mathbf{K}+\mathbf{q}) \cdot \mathbf{r}^{l}\right]
$$

vanishes unless $\mathbf{K}+\mathbf{q}=\mathbf{S}$, where $\mathbf{S}$ is a reciprocal-lattice vector. Eliminating $\mathbf{K}$ from Eq. (4), we obtain

$$
A_{\mathrm{ds}}=i f_{0}(\mathbf{S}+\mathbf{q}) \cdot \mathbf{Q}(-\mathbf{q})
$$

and the corresponding scattering intensity is

$$
I_{\mathrm{ds}}(\mathbf{S}+\mathbf{q})=f_{0}^{2}|(\mathbf{S}+\mathbf{q}) \cdot \mathbf{Q}(-\mathbf{q})|^{2} .
$$

Hence we may find the diffuse scattering intensity at a point in reciprocal space at a distance $|\mathbf{q}|$ from the reciprocal-lattice site $\mathbf{S}$, once we know the appropriate Fourier amplitude $\mathbf{Q}(-\mathbf{q})$. Kanzaki has pointed out that the diffuse scattering intensity will be largest near reciprocal-lattice points, i.e., in the region where $q \sim 0$. Hence we have chosen to investigate the diffuse scattering in this region only. In the limit as $\mathbf{q} \sim 0$, Eq. (6) becomes

$$
I_{\mathrm{ds}}=f_{0}{ }^{2}|\mathbf{S} \cdot \mathbf{Q}(\mathbf{q} \sim 0)|^{2} .
$$

This gives the diffuse scattering intensity for one defect, and in the limit of low concentration, the appropriate scattering for a random array of defects is given by

$$
I_{\mathrm{ds}}=n f_{0}^{2}|\mathbf{S} \cdot \mathbf{Q}(\mathbf{q} \sim 0)|^{2},
$$

where $n$ is the number of defects. This means that if the asymptotic form of $\mathbf{Q}(\mathbf{q})$ is known for a given type of defect one can predict isointensity profiles about $\mathbf{S}$. These profiles are in principle directly comparable with experiment.

The derivation of the asymptotic expression for $\mathbf{Q}(\mathbf{q})$ where $\mathbf{q} \sim 0$ has been given by Kanzaki ${ }^{1}$ and by ourselves. ${ }^{5}$ For a defect of cubic symmetry in a cubic host lattice, if we take as our coordinate system Cartesian axes along the $\langle 100\rangle$ directions, the three components of $\mathbf{Q}$ are equal and are given by

$$
Q_{\alpha}=C_{s}\left[k_{\alpha} / P(\mathbf{k})\right]\left(E k^{4}+F k_{\alpha}{ }^{2} k^{2}+H k_{\beta}{ }^{2} k_{\gamma}^{2}\right),
$$

where $\alpha \neq \beta \neq \gamma \neq \alpha$, and $\mathbf{k} \equiv \mathbf{q} a$, where $a$ is half of a cubic unit-cell edge; $\alpha, \beta$, and $\gamma$ refer to the components along the three axes, and $P(\mathbf{k})$ is given by

$$
\begin{array}{r}
P(\mathbf{k})=D k^{6}+B k^{2}\left(k_{\alpha}{ }^{2} k_{\beta}{ }^{2}+k_{\beta}{ }^{2} k_{\gamma}{ }^{2}+k_{\gamma}{ }^{2} k_{\alpha}{ }^{2}\right) \\
+A k_{\alpha}{ }^{2} k_{\beta}{ }^{2} k_{\gamma}{ }^{2},
\end{array}
$$

where, again, $\alpha \neq \beta \neq \gamma \neq \alpha$.

The constants in the bracketed term in Eq. (9) and in the equation for $P(\mathbf{k})$ above may be expressed in terms of either the direct-space force constants or the elastic constants of the host lattice. In terms of the latter, they are given by

$$
\begin{aligned}
& A=\left(C_{11}-C_{12}-2 C_{44}\right)\left[\left(C_{11}-C_{44}\right)\left(C_{11}+C_{12}\right)\right. \\
& \left.\quad-2\left(C_{12}+C_{44}\right)^{2}\right], \\
& B=C_{44}\left(C_{11}+C_{12}\right)\left(C_{11}-C_{12}-2 C_{44}\right), \\
& D=C_{11} C_{44}{ }^{2}, \\
& E=C_{44}\left(C_{11}-C_{12}-C_{44}\right), \\
& F=C_{44}\left(C_{12}+2 C_{44}-C_{11}\right), \\
& H=\left(C_{11}-C_{12}-2 C_{44}\right)^{2},
\end{aligned}
$$

where the $C_{i j}$ are the three independent elastic constants for a cubic lattice.

The coefficient $C_{s}$ in Eq. (9) may be directly related to the strength parameter $G$ described in Ref. 5 as follows:

$$
C_{s}=-i G a / v \text {. }
$$

Here, $v$ is the atomic volume for the lattice and $G$ is the strength parameter given by a prescription due to Hardy, ${ }^{9}$

$$
G_{\alpha \beta}=\sum_{l} F_{\alpha}^{l} r_{\beta}^{l},
$$

where $F_{\alpha}{ }^{l}$ is the $\alpha$ th component of the direct-space force exerted by the defect on the $l$ th atom. These forces are obtained by a lattice static calculation.

Although $G$ is normally a tensor, for a cubic defect the off-diagonal terms vanish and all of the diagonal elements are identical.

For a double-force defect along [110], the Fourier components $\mathbf{Q}(\mathbf{q} \sim 0)$ are given by

$$
\begin{aligned}
Q_{\alpha}= & {\left[C_{d} / P(\mathbf{k})\right] } \\
& \times\left[\left(E^{\prime} k^{4}+V^{\prime} k^{2} k_{\alpha}{ }^{2}+J k^{2} k_{\beta}{ }^{2}+M k_{\beta}{ }^{2} k_{\gamma}{ }^{2}\right) k_{\alpha}\right. \\
& \left.+\left(E^{\prime} k^{4}+T k^{2} k_{\alpha}^{2}+H^{\prime} k_{\beta}{ }^{2} k_{\gamma}{ }^{2}+W k_{\alpha}{ }^{2} k_{\gamma}{ }^{2}\right) k_{\beta}\right], \\
Q_{\beta}= & {\left[C_{d} / P(\mathbf{k})\right] } \\
& \times\left[\left(E^{\prime} k^{4}+V^{\prime} k^{2} k_{\beta}{ }^{2}+J k^{2} k_{\alpha}{ }^{2}+M k_{\alpha}{ }^{2} k_{\gamma}{ }^{2}\right) k_{\beta}\right. \\
& \left.+\left(E^{\prime} k^{4}+T k^{2} k_{\beta}{ }^{2}+H^{\prime} k_{\alpha}{ }^{2} k_{\gamma}{ }^{2}+W k_{\beta}{ }^{2} k_{\gamma}{ }^{2}\right) k_{\alpha}\right], \\
Q_{\gamma}= & {\left[C_{d} / P(\mathbf{k})\right]\left\{\left[2 W k_{\alpha}{ }^{2} k_{\beta}{ }^{2}+J\left(k^{4}-k^{2} k_{\gamma}{ }^{2}\right)\right] k_{\gamma}\right.} \\
& \left.+\left[W\left(k^{2}-k_{\gamma}{ }^{2}\right)+2 J k^{2}\right] k_{\alpha} k_{\beta} k_{\gamma}\right\},
\end{aligned}
$$

where $\alpha \neq \beta \neq \gamma \neq \alpha$. Here,

$$
C_{d}=-i G^{\prime} a / v
$$

where $G^{\prime}$ is obtained by the prescription given in Eq. (13).

Since $\alpha$ and $\beta$ lie along [100] and [010], respectively, they are equivalent orthogonal axes in the plane of the double force. Here $\gamma$ lies along [001] and is perpendicular to $\alpha, \beta$ and the double force. The constants in

\footnotetext{
${ }^{9}$ J. R. Hardy, J. Phys. Chem. Solids 29, 2009 (1968).
} 
FIG. 6. Isointensity profiles near an $(S, S, S)$ reciprocal-lattice point (A) for a cubic defect, and (B) for a doubleforce defect along $\langle 110\rangle$, in $\mathrm{Cu}$. Solid lines, in the $[1 \overline{1} 0]$ plane; dashed lines, in the $[10 \overline{1}]$ plane.

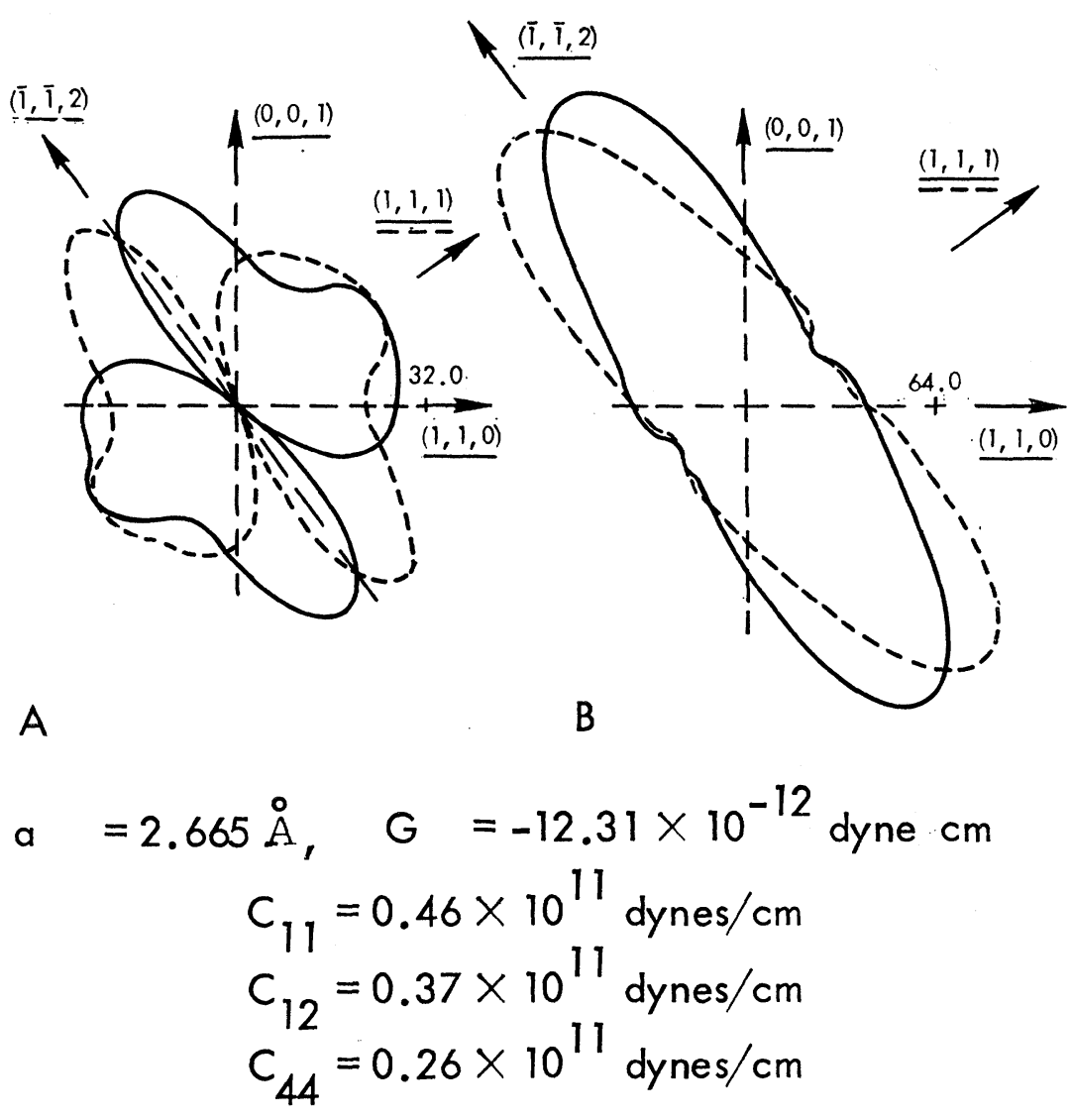

Eq. (14) are given by

$$
\begin{aligned}
E^{\prime} & =C_{44} C_{11}, \\
V^{\prime} & =-C_{44}\left(C_{11}-C_{44}\right), \\
H^{\prime} & =\left(C_{11}{ }^{2}-C_{12}{ }^{2}\right)-2 C_{44}\left(C_{11}+C_{12}\right), \\
J & =-C_{44}\left(C_{12}+C_{44}\right), \\
W & =\left(C_{12}+C_{44}\right)\left(C_{12}-C_{11}+2 C_{44}\right), \\
M & =-\left(C_{12}+C_{44}\right)\left(C_{11}-C_{44}\right), \\
T & =-C_{44}\left(C_{11}+C_{12}\right) .
\end{aligned}
$$

Since the strength parameters $G^{\prime}$ for the double forces are not known, we have arbitrarily set them equal to the corresponding parameter $G$ for the cubic defect in each material. This allows us to gain some insight into the relative scattering intensities produced by two different types of defects having the same strengths. When actual strength parameters for the double forces are obtained, the corresponding intensities may readily be obtained by appropriately scaling our results.

\section{APPLICATIONS}

We have calculated isointensity profiles for diffuse $\mathrm{x}$-ray scattering induced by cubic and double-force defects in $\mathrm{Al}, \mathrm{Cu}, \mathrm{K}, \mathrm{Na}, \mathrm{Li}$, and Kanzaki's model lattice, for which $C_{11}=2 C_{12}=2 C_{44}$. The resultant profiles have been plotted in various symmetry planes about $(S, 0,0)$-, $(S, S, 0)$-, and $(S, S, S)$-type reciprocallattice points for each defect class in each material. Since the diffuse scattering intensity falls off as $1 /|q|^{2}$ along any given direction, we can write

where

$$
I_{\mathrm{ds}}=\left(f_{0}^{2} /|\mathbf{q}|^{2}\right) F_{s}(\mathbf{q} /|\mathbf{q}|) \text {, }
$$

$$
F_{s}(\mathbf{q} /|\mathbf{q}|) \equiv|\mathbf{q}|^{2}|\mathbf{S} \cdot \mathbf{Q}(\mathbf{q} \sim 0)|^{2}
$$

and is a function of direction only, for a given reciprocallattice point $\mathbf{S}$. The surface in reciprocal space, on which the intensity of diffuse scattering is $I_{\mathrm{ds}}$, can be found by plotting

$$
|\mathbf{q}|=\frac{f_{0}}{\sqrt{ } I_{\mathrm{ds}}}\left[F_{s}(\mathbf{q} /|\mathbf{q}|)\right]^{1 / 2}
$$

in all directions about the reciprocal-lattice point $\mathbf{S}$. For convenience, we have set $f_{0} / \sqrt{ } I_{\mathrm{ds}}=1$ in our calculations.

The coefficients $C_{s}$ were calculated in each case from the direct-space forces previously calculated for vacancies in $\mathrm{Al}$ and $\mathrm{Cu}^{10}$ For the remaining materials, including the model lattice, the cubic defect was repre-

\footnotetext{
${ }^{10}$ R. Bullough and J. R. Hardy, Phil. Mag. 17, 833 (1968).
} 


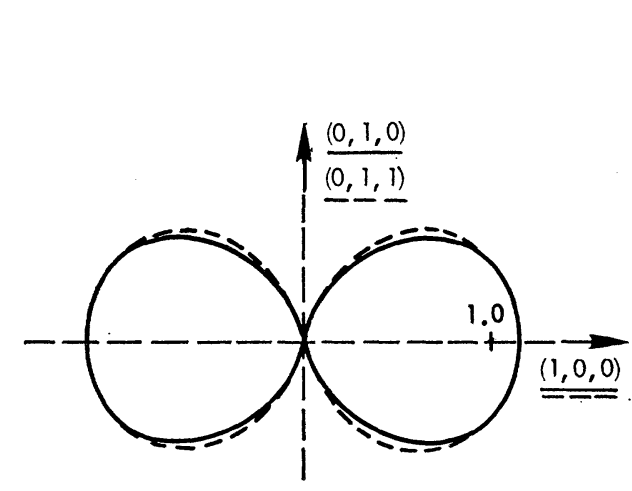

A

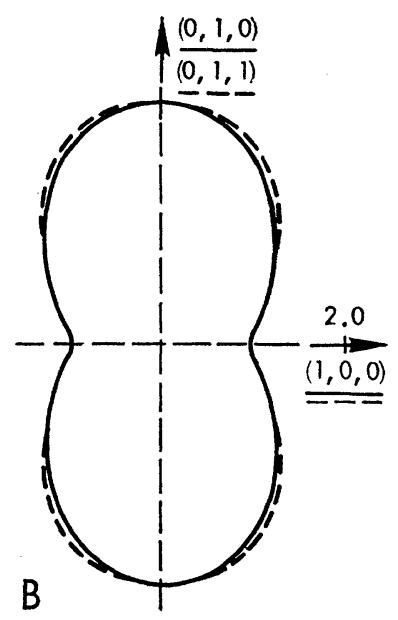

B
FIG. 7. Isointensity profiles near an $(S, 0,0)$ reciprocal-lattice point (A) for a cubic defect, and (B) for a doubleforce defect along $\langle 110\rangle$, in $\mathrm{Al}$. Solid lines, in the [001] plane; dashed lines in the [011] plane.

$$
\begin{aligned}
a=2.02 \AA, \quad G=-3.308 \times 10^{-12} \text { dyne } \mathrm{cm} \\
C_{11}=1.08 \times 10^{12} \text { dynes } / \mathrm{cm} \\
C_{12}=0.62 \times 10^{12} \text { dynes } / \mathrm{cm} \\
C_{44}=0.28 \times 10^{12} \text { dynes } / \mathrm{cm}
\end{aligned}
$$

sented by a superposition of three orthogonal double forces at the origin. The axes of these three double forces were taken along the $\langle 100\rangle$ crystallographic directions and each component of the force array was taken to be $10^{-4} \mathrm{dyn}$. (This is a typical value for the systems we have considered.) For the single double force, we arbitrarily set $G^{\prime}=G$. This was done for convenience, but it is obvious that the actual magnitudes of $G$ and $G^{\prime}$ have no effect on the form of our results, since they appear in our equations simply as scaling factors.

When we calculated the isointensity profiles for the single double force, we allowed for the fact that there are six equivalent orientations for the [110] double force. We assumed that the defects were equally distributed over the six orientations, and thus in making our calculations we calculate the diffuse intensity contribution from each of these six configurations separately and then average the results. Our calculations were performed in part on the CDC-3600 computer at Lawrence Radiation Laboratory, Livermore, Calif., and in part on the IBM-360/65 at the University of Nebraska.

Figure 1 shows the isointensity profiles in the vicinity of an $(S, 0,0)$-type reciprocal-lattice point in the model lattice, for a cubic defect and a single double-force defect, respectively. Figures 2 and 3 show corresponding isointensity profiles about $(S, S, 0)$ - and $(S, S, S)$-type reciprocal-lattice points, respectively, in the model lattice. Each diagram contains profiles for two planes which have a reciprocal-lattice point at the origin and intersect along the vector $\mathbf{S}$.

Figures 4-6 are the corresponding profiles for $\mathrm{Cu}$, and Figs. 7-9 are those for Al. The profiles for $\mathrm{K}$ and $\mathrm{Li}$ are shown in Figs. 10-15. No profiles are given for $\mathrm{Na}$, since they are almost identical to those for $\mathrm{K}$, both in shape and order of magnitude.

\section{DISCUSSION}

Our results for the model lattice are the same as those given by Kanzaki ${ }^{1}$ except for the profile about the $(S, S, S)$ type of point for the single double force. In this case, Kanzaki's results show an outward bulge about the $\langle 111\rangle$ direction which is completely absent from our results. We have checked our results for this particular case very closely and we feel that there must be some error in Kanzaki's original calculation.

One of the most important results of this work is that our results confirm Kanzaki's conclusion that cubic defects produce isointensity profiles which are lemniscates, while single double-force defects (such as "dumbbell" interstitials or divacancies) produce ellipsoidal profiles. This fact is significant in that the shape of the x-ray diffuse pattern near a reciprocal-lattice point should provide a good deal of information concerning the symmetry of point defects present in a specimen.

Another interesting effect, not revealed by Kanzaki's calculations, is the way in which the anisotropy of the 
FIG. 8. Isointensity profiles near an $(S, S, 0)$ reciprocal-lattice point (A) for a cubic defect, and (B) for a double-force defect along $\langle 110\rangle$, in Al. Solid lines, in the [001] plane; dashed lines, in the $[110]$ plane.

FIG. 9. Isointensity profiles near an $(S, S, S)$ reciprocal-lattice point (A) for a cubic defect, and (B) for a doubleforce defect along $\langle 110\rangle$, in Al. Solid lines, in the [110] plane; dashed lines, in the [10ī] plane.

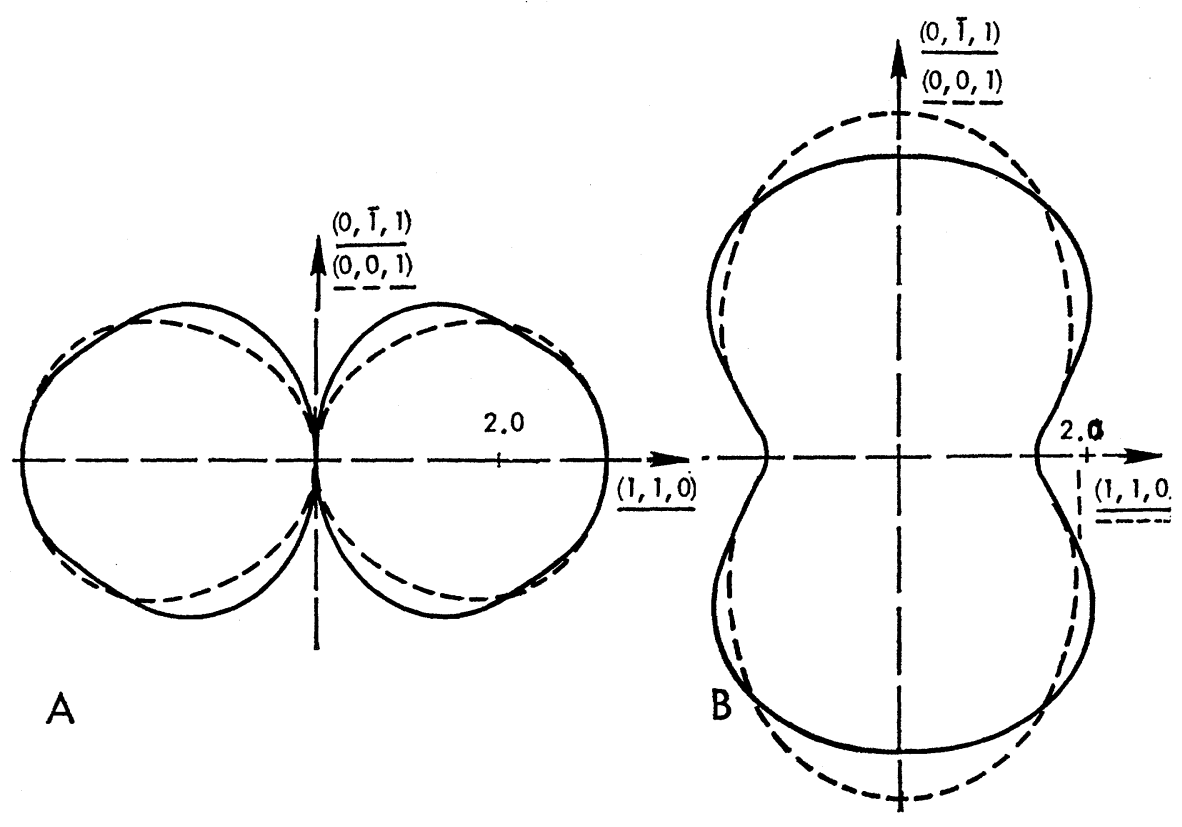

$$
\begin{aligned}
a=2.02 \AA, \quad G=-3.308 \times 10^{-12} \text { dyne } \mathrm{cm} \\
C_{11}=1.08 \times 10^{12} \text { dynes } / \mathrm{cm} \\
C_{12}=0.62 \times 10^{12} \text { dynes } / \mathrm{cm} \\
C_{44}=0.28 \times 10^{12} \text { dynes } / \mathrm{cm}
\end{aligned}
$$

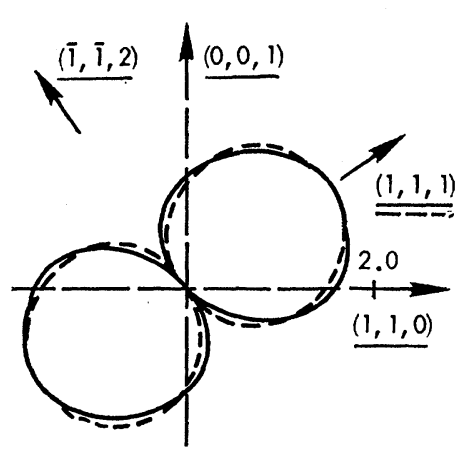

A

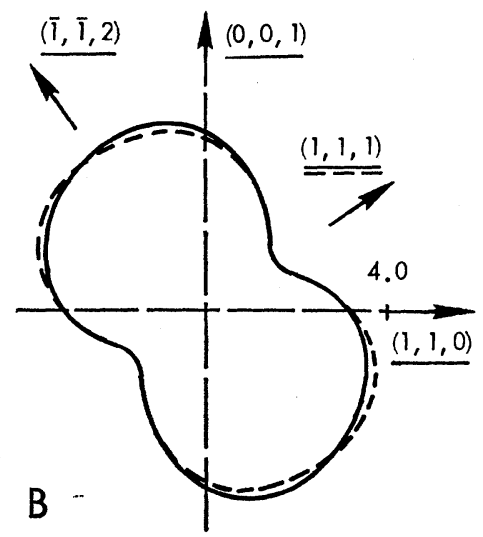

$a=2.02 \AA, \quad G=-3.308 \times 10^{-12}$ dyne $\mathrm{cm}$

$C_{11}=1.08 \times 10^{12}$ dynes $/ \mathrm{cm}$

$C_{12}=0.62 \times 10^{12}$ dynes $/ \mathrm{cm}$ $C_{44}=0.28 \times 10^{12}$ dynes $/ \mathrm{cm}$ 


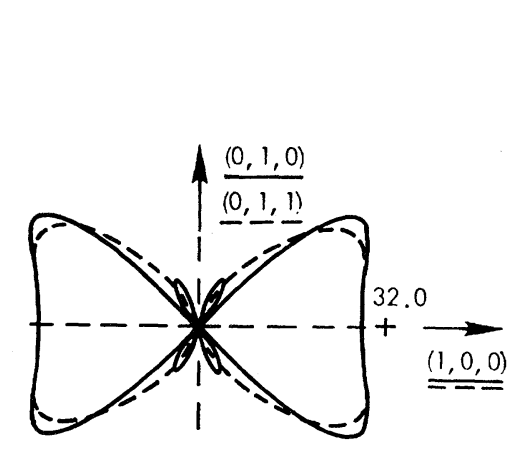

A

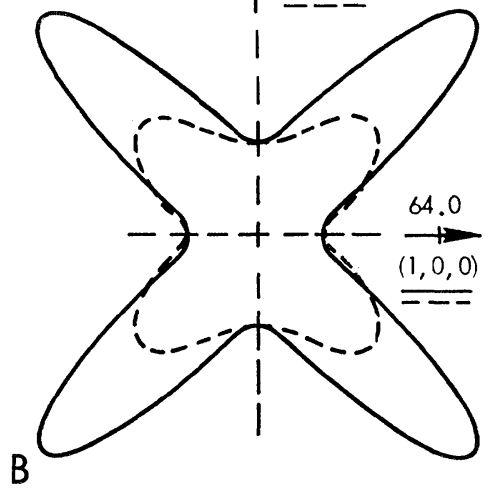

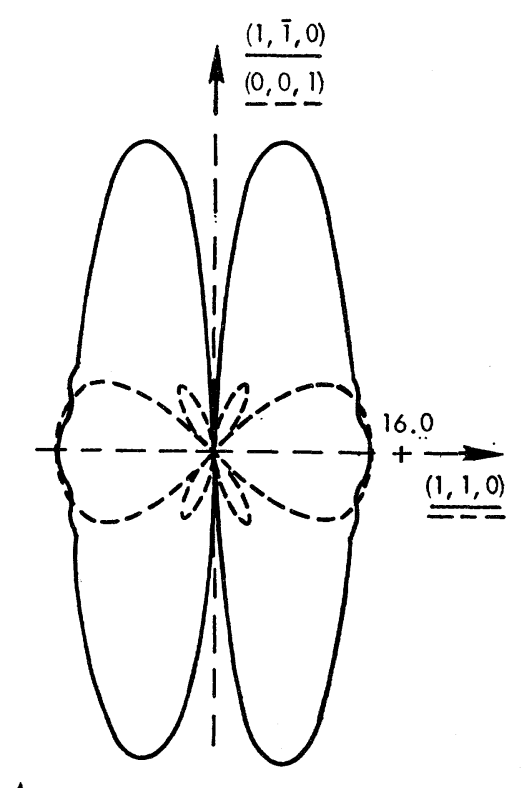

A

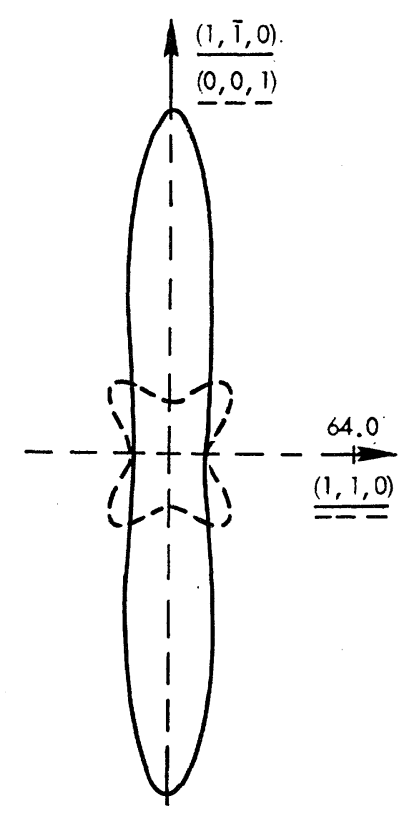

$$
\begin{aligned}
a=1.75 \AA, \quad G=-8.08 \times 10^{-12} \text { dyne } \mathrm{cm} \\
C_{11}=0.1306 \times 10^{12} \text { dynes } / \mathrm{cm} \\
C_{12}=0.1102 \times 10^{12} \text { dynes } / \mathrm{cm} \\
C_{44}=0.088 \times 10^{12} \text { dynes } / \mathrm{cm}
\end{aligned}
$$

FIG. 10. Isointensity profiles near an $(S, 0,0)$ reciprocal-lattice point $(\mathrm{A})$ for a cubic defect, and (B) for a doubleforce defect along $\langle 110\rangle$, in K. Solid lines, in the [001] plane; dashed lines, in the [011] plane.

FIG. 11. Isointensity profiles near an $(S, S, 0)$ reciprocal-lattice point $(\mathrm{A})$ for a cubic defect, and (B) for a doubleforce defect along $\langle 110\rangle$, in K. Solid lines, in the [001] plane; dashed lines, in the $[1 \overline{1} 0]$ plane. 
FIG. 12. Isointensity profiles near an $(S, S, S)$ reciprocal-lattice point (A) for a cubic defect, and (B) for a doubleforce defect along $\langle 110\rangle$, in $\mathrm{K}$. Solid lines, in the [110] plane; dashed lines, in the [10i] $]$ plane.

FIG. 13. Isointensity profiles near an $(S, 0,0)$ reciprocal-lattice point $(\mathrm{A})$ for a cubic defect, and (B) for a doubleforce defect along $\langle 110\rangle$, in Li. Solid lines, in the [001] plane; dashed lines in the [011] plane.
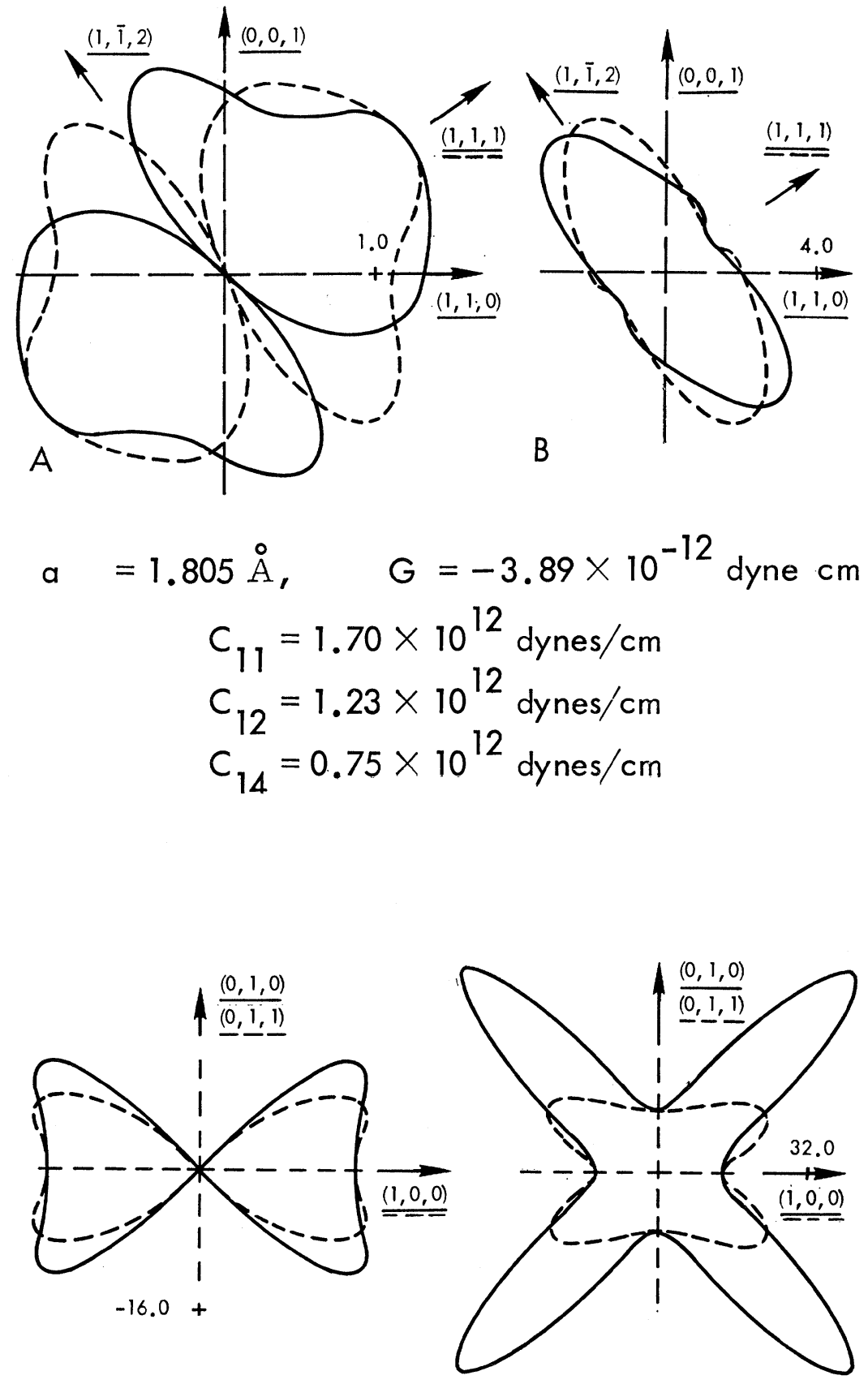

A.

B.

$$
\begin{aligned}
a=1.75 \AA & \quad G=-8.08 \times 10^{-12} \text { dyne } \mathrm{cm} \\
C_{11} & =0.1306 \times 10^{12} \text { dynes } / \mathrm{cm} \\
C_{12} & =0.1102 \times 10^{12} \text { dynes } / \mathrm{cm} \\
C_{44} & =0.088 \times 10^{12} \text { dynes } / \mathrm{cm}
\end{aligned}
$$



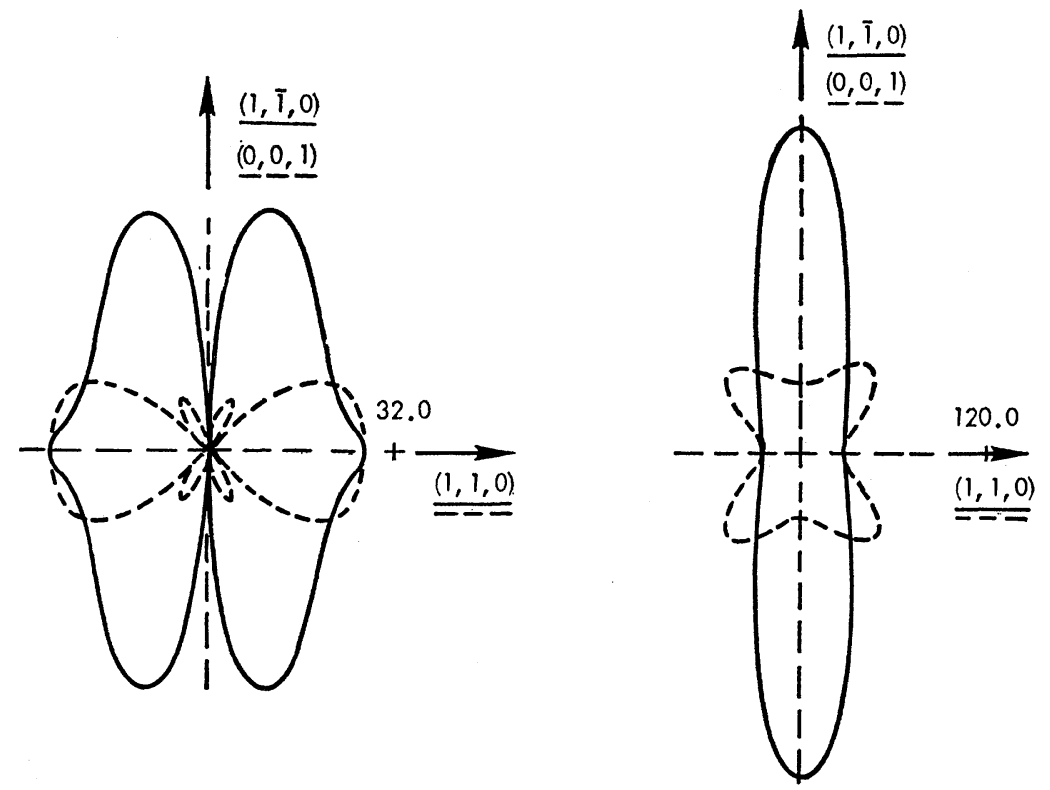

FIG. 14. Isointensity profiles near an $(S, S, 0)$ reciprocal-lattice point $(\mathrm{A})$ for a cubic defect, and (B) for a doubleforce defect along $\langle 110\rangle$, in Li. Solid lines, in the [001] plane; dashed lines in the [110] plane.

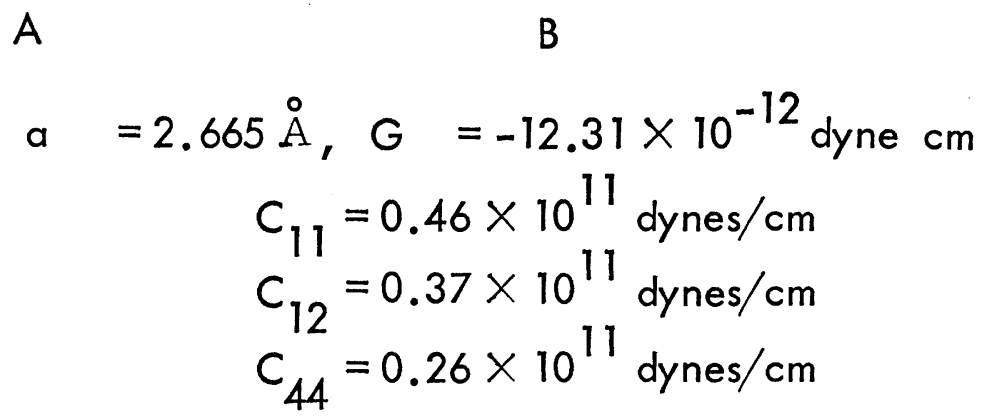

crystal affects the intensity profiles. The effect is most evident in the results for $\mathrm{Al}$, which is nearly isotropic. For a given type of defect, the profiles in the two symmetry planes about any given type of reciprocallattice point are almost identical. In addition, the profiles about all three types of reciprocal-lattice points are very similar both for the cubic defect and the double force. Corresponding comparisons for the other materials do not reveal such similarities. If the $\mathrm{Al}$ profiles are used as a standard and compared with the corresponding profiles for the other crystals, striking variations can be observed arising from anisotropy. The two lobes of the lemniscate-type profiles, which are nearly spherical for $\mathrm{Al}$, undergo drastic changes of shape for the other materials, generally flattening or even becoming concave at the ends farthest from the origin. Often smaller lobes are seen to arise near the origin.

It is also apparent from the scale factors, indicated on each figure, that the single double forces give rise to directional "spikes" which are of much greater intensity than any feature of the corresponding profile for the cubic defects. All of the effects that we have pointed out should be of some importance in determining the type and relative number of defects present in a material under any given conditions. We have only considered cubic and double-force defects here, but the technique should be readily applicable to more complex types of defect aggregates.

Finally, we must point our once again that the present work only applies to regions in reciprocal space which are very close to reciprocal-lattice points, where the diffuse scattering intensity is highest.

\section{SUMMARY}

We have repeated the calculations made by Kanzaki ${ }^{1}$ using the same technique, which makes use of the method of lattice statics in the asymptotic limit as $q \sim 0$. This technique allows the calculation of the isointensity contours appropriate to defect-induced $\mathrm{x}$-ray diffuse scattering. We have obtained results for cubic and double-force defects in $\mathrm{Al}, \mathrm{Cu}, \mathrm{Na}, \mathrm{K}, \mathrm{Li}$, and the model lattice treated by Kanzaki, for which $C_{11}=2 C_{12}=2 C_{44}$. We have given isointensity profiles in certain high-symmetry planes about $(S, 0,0),(S, S, 0)$, and $(S, S, S)$ types of reciprocal-lattice points.

We agree with Kanzaki's general conclusions that cubic defects produce isointensity profiles which have a 
FIG. 15. Isointensity profiles near an $(S, S, S)$ reciprocal-lattice point (A) for a cubic defect, and (B) for a doubleforce defect along $\langle 110\rangle$, in $\mathrm{Li}$. Solid lines, in the $[1 \overline{1} 0]$ plane; dashed lines, in the $[10 \overline{1}]$ plane.
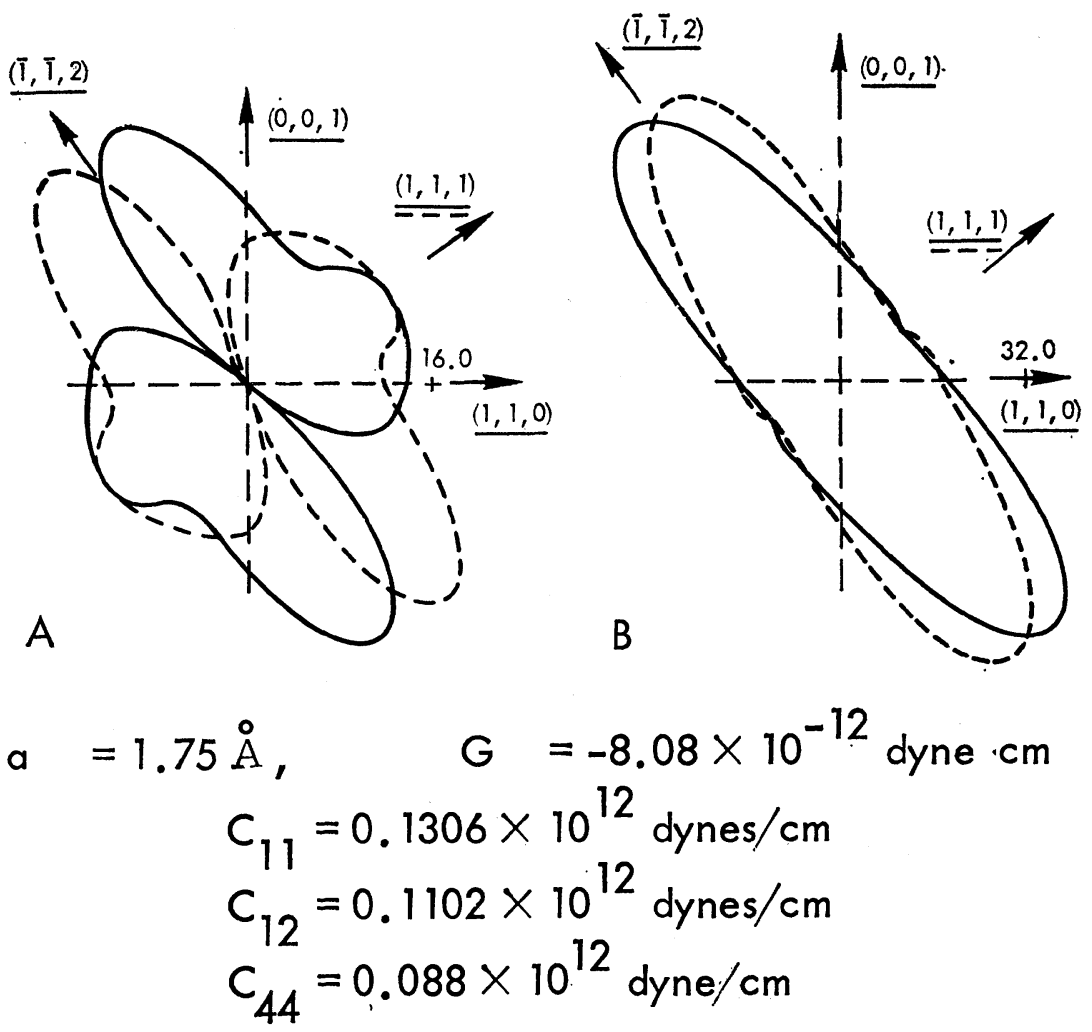

lemniscate shape, while double-force defects produce ellipsoidal profiles. The profiles obtained for the model lattice treated by Kanzaki agree with his profiles except for those about the $(S, S, S)$ type of point, produced by a double-force defect.

The method of lattice statics is particularly suited to computations of the type described in this paper. The reason for this is that lattice statics gives us, directly, the Fourier transform of the defect strain field, and it is this quantity which is essentially measured by the diffuse-scattering intensity. This procedure is obviously very much superior to that in which one determines the direct-space displacements and then attempts to compute the form factors for the distorted lattice by brute force. Lattice statics provides the essential simplification of eliminating the cumbersome intermediate step involved in calculating the displacements themselves. Moreover, in the asymptotic limit, $q \sim 0$, the equations of lattice statics as used in the present paper, go naturally over into the equations of continuum elasticity.

We must point out again that we have neglected terms in the diffuse-scattering intensity which are related to the size and scattering factor of the defect. We have, essentially, treated the simplest term in the intensity expansion in order to demonstrate the usefulness of the method of lattice statics in this general type of calculation. Evaluation of succeeding terms will be more difficult in practice but will make use of the Fourier amplitudes of the imperfect lattice in exactly the same manner as we have used them in the present case.

The results of the present paper are definitive for the problem which is considered, in that the forms of the isointensity profiles are essentially determined by the elastic constants. Subsequent work will only modify the present results by simple numerical scaling factors as the true magnitudes of $G$ and $G^{\prime}$ become known. 
X-Ray Determination of Phonon Dispersion in Vanadium, R. Colella and B. W. Batterman [Phys. Rev. B 1, 3913 (1970)]. Reference 13 (p. 3915, left column, line 10) should be read as 15. Reference 15 (p. 3915, left column, line 28) should be read as 7. Reference 17 (p. 3915, right column, line 2) should be read as 13 .

Calculations of the Intensity of X-Ray Diffuse Scattering Produced by Point Defects in Cubic Metals, John W. Flocken and John R. Hardy [Phys. Rev. B 1, 3913 (1970)]. Reference 13 (p. 3915, and figure captions have become interchanged. They should be corrected as follows.

Figure 6 and 12: The caption of Fig. 6 should be retained but the figures and the accompanying data should be replaced by those shown in Fig. 12 .

Likewise, the figures and data of Fig. 12 should be replaced by those of Fig. 6 .

Figures 11 and 14: The caption of Fig. 11 should be retained but the figures and data should be replaced by those of Fig. 14. Likewise, the caption of Fig. 14 applies to the figures and data shown in Fig. 11.

We are much indebted to Dr. G. Wolfram for pointing out these discrepancies. 